\title{
Interaction between the divacancy and hydrogen in silicon: Observation of fast and slow kinetics
}

Cite as: J. Appl. Phys. 124, 085706 (2018); https://doi.org/10.1063/1.5037310

Submitted: 23 April 2018 . Accepted: 10 August 2018 . Published Online: 29 August 2018

I. L. Kolevatov (D), B. G. Svensson, and E. V. Monakhov
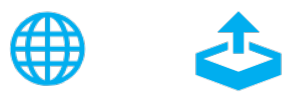

\section{ARTICLES YOU MAY BE INTERESTED IN}

Impact ionization in $\beta-\mathrm{Ga}_{2} \mathrm{O}_{3}$

Journal of Applied Physics 124, 085707 (2018); https://doi.org/10.1063/1.5034120

Defects responsible for charge carrier removal and correlation with deep level introduction in irradiated $\beta-\mathrm{Ca}_{2} \mathrm{O}_{3}$

Applied Physics Letters 113, 092102 (2018); https://doi.org/10.1063/1.5049130

Tutorial: Junction spectroscopy techniques and deep-level defects in semiconductors Journal of Applied Physics 123, 161559 (2018); https://doi.org/10.1063/1.5011327

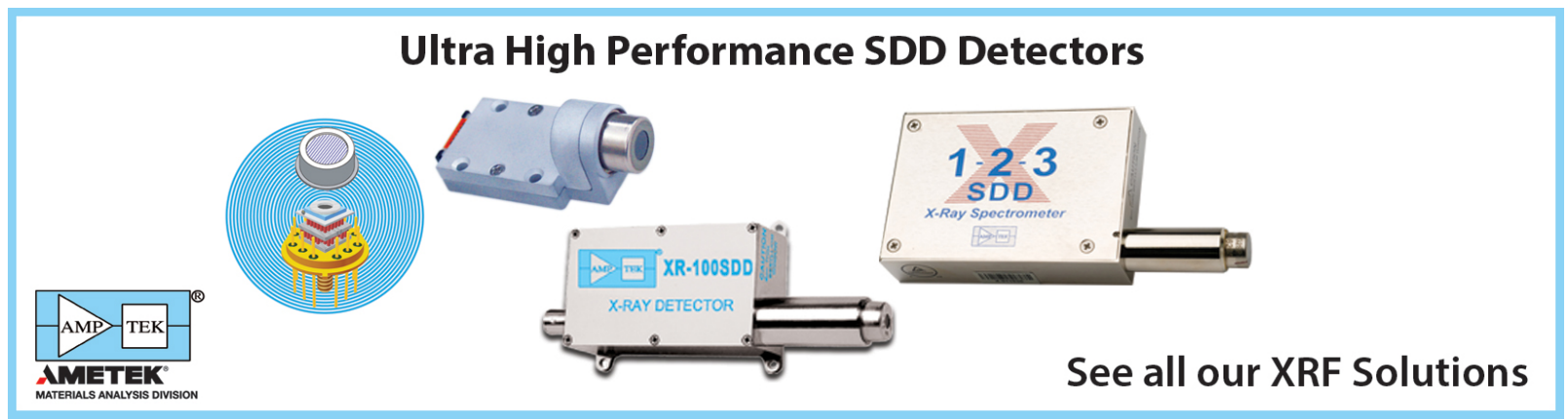




\title{
Interaction between the divacancy and hydrogen in silicon: Observation of fast and slow kinetics
}

\author{
I. L. Kolevatov, B. G. Svensson, and E. V. Monakhov \\ University of Oslo, Department of Physics, Center for Materials Science and Nanotechnology, P.O. Box 1048, \\ Blindern, Oslo, Norway
}

(Received 23 April 2018; accepted 10 August 2018; published online 29 August 2018)

\begin{abstract}
The divacancy $\left(V_{2}\right)$ is one of the fundamental defects in silicon. However, the interaction of $V_{2}$ with hydrogen is still not fully understood. In the present work, deep level transient spectroscopy (DLTS) results on hydrogen-assisted annealing of $V_{2}$ are presented. $\mathrm{H}^{+}$ions were implanted with multiple energies into n-type Czochralski-grown samples, yielding uniform (box-like) concentration-versus-depth profiles of $V_{2}$ and hydrogen in the region probed by the DLTS measurements. The evolution kinetics of $V_{2}$ reveals two distinct processes: (i) a fast one attributed to dissociation of phosphorus-hydrogen pairs and reaction with highly mobile atomic $\mathrm{H}_{i}$ and (ii) a slow one whose origin is not identified yet. During the slow process, we observe the formation of a hydrogen-related electronic state, labeled $E 5^{*}$ and positioned $\sim 0.42 \mathrm{eV}$ below the conduction band edge. The growth of $E 5^{*}$ displays a close one-to-one proportionality with the loss of a $V_{2}$-related DLTS peak, presumably due to $V_{2} \mathrm{H}$, overlapping with that of the single negatively charged $V_{2}$. Published by AIP Publishing. https://doi.org/10.1063/1.5037310
\end{abstract}

\section{INTRODUCTION}

The divacancy $\left(V_{2}\right)$ is perhaps the most fundamental intrinsic defect in silicon stable at room temperature. It has four charge states: positive $(+)$, neutral $(0)$, singly negative $(-)$, and doubly negative $(=) .{ }^{1}$ Despite a large amount of studies, there is still some controversy regarding its annealing mechanisms in different types of silicon materials. The $V_{2}$ dissociation prevails in oxygen-lean float zone silicon (Fz-Si), while the diffusion mechanism dominates in oxygen-rich Czochralski-grown silicon $(\mathrm{Cz}-\mathrm{Si})$ where $V_{2}$ can be trapped by interstitial oxygen atoms $\left(\mathrm{O}_{i}\right)$, forming divacancy-oxygen complexes $\left(V_{2} \mathrm{O}\right){ }^{1}$

However, these two mechanisms become less important in hydrogen-rich silicon materials. The presence of hydrogen significantly reduces the annealing temperature of $V_{2}{ }^{2}$ The interaction of $V_{2}$ with hydrogen is more complex and considerably less studied than that with $\mathrm{O}_{i}$. EPR and Fourier transform infrared spectroscopy (FTIR) signals of $V_{2} \mathrm{H}$ have been identified in hydrogen-implanted samples. ${ }^{3}$ However, the identification of electronic levels related to $V_{2} \mathrm{H}_{n}$ with $\mathrm{n}=1$, 3 , or 5 is still controversial. On the basis of their atomic structure (Ref. 4), these complexes are anticipated to have three charge states: $(+),(0)$, and $(-)$, and theory predicts that the donor $(0 /+)$ and acceptor $(-/ 0)$ levels are located close to those of $V_{2}(0 /+)$ and $V_{2}(-/ 0)$, respectively. Experimentally, this prediction is still not conclusively confirmed. For example, in Deep Level Transient Spectroscopy (DLTS) studies of $\mathrm{H}^{+}$-implanted n-type samples, the amplitude of the DLTS peak assigned to $V_{2}(-/ 0)$ exceeds that of the double-acceptor state $V_{2}(=/-)$, by almost a factor of two in some cases. ${ }^{5-9}$ This contradicts the expectations since an isolated $V_{2}$ should exhibit DLTS peaks of $V_{2}(-/ 0)$ and $V_{2}(=/-)$ with equal amplitudes, which is indeed observed after electron irradiation. $^{2,8-10}$ It is known that the DLTS peaks of $V_{2}(-/ 0)$ and
$V_{2}(=/-)$ may differ in amplitude in the case of heavy ion implantation. ${ }^{11}$ However, for $\mathrm{H}^{+}$ions, this effect is weak and amounts only to around $10 \% .^{11}$ It has been suggested, hence, that the enhancement of the $V_{2}(-/ 0)$ DLTS peak is due to a contribution from $V_{2} \mathrm{H}(-/ 0)$, overlapping with $V_{2}(-/ 0)$, but studies of the annealing kinetics did not result in a fully conclusive identification of $V_{2} \mathrm{H}^{6,7}$

The general picture of the hydrogen interaction with $V_{2}$ becomes even more complex when hydrogen is introduced by other techniques than implantation, such as hydrogenation from hydrogen-plasma (Ref. 2) or chemical solution (Ref. $8)$. In these studies, $V_{2}$ was generated by $\mathrm{MeV}$ electron irradiation, and the $V_{2}(=/-)$ and $V_{2}(-/ 0)$ DLTS peaks exhibited almost identical amplitudes not only after the irradiation but also during the subsequent hydrogen-assisted annealing. This rules out the formation of an overlapping $V_{2} \mathrm{H}$ level in these samples. It has been realized then that diatomic hydrogen $\left(\mathrm{H}_{2}\right)$, or hydrogen dimers, can play an important role in the $V_{2}$ annealing. Thus, the absence of a $V_{2} \mathrm{H}$ level in these studies has been explained by the interaction $V_{2}+\mathrm{H}_{2} \rightarrow V_{2} \mathrm{H}_{2}$. The formation of $V_{2} \mathrm{H}_{2}$, however, has not been identified via the emergence of an electronic level.

It is interesting to note that theory predicts that $V_{2} \mathrm{H}_{2}$ is electrically active as well. ${ }^{4}$ It is expected that the acceptor state $V_{2} \mathrm{H}_{2}(-/ 0)$ occurs at $\sim E_{c}-0.32 \mathrm{eV}$ ( $E_{c}$ being the edge of the conduction band). This predicted level position of $V_{2} \mathrm{H}_{2}(-/ 0)$ coincides with that of $\mathrm{VOH}(-/ 0)$, which is also located at about $E_{c}-0.32 \mathrm{eV}$ (Ref. 8 and references therein), and a strong overlap between $V_{2} \mathrm{H}_{2}(-/ 0)$ and $V \mathrm{OH}(-/ 0)$ in DLTS spectra can be expected. $V \mathrm{OH}$ is one of the most dominant complexes in irradiated and hydrogen-enriched silicon, with a concentration significantly exceeding that of $V_{2} \mathrm{H}_{2}$. This makes the detection of $V_{2} \mathrm{H}_{2}(-/ 0)$ challenging. With respect to the donor state $V_{2} \mathrm{H}_{2}(0 /+)$, theory does not predict any level in the bandgap. ${ }^{4}$ 
In the present work, we report on the evolution of $V_{2}$ and associated complexes upon heat treatments of $\mathrm{H}^{+}$-implanted samples using high-resolution DLTS analysis. The $\mathrm{H}^{+}$-implantation was performed with multiple energies to form a uniform box-like profile of $V_{2}$ and hydrogen, facilitating the data analysis and interpretation. Upon annealing, a new hydrogen-related level, labeled $E 5^{*}$, is revealed. Its formation kinetics is slower than that of other defect reactions involving $\mathrm{H}_{i}$, and it anticorrelates in a one-to-one ratio with the state commonly ascribed to $V_{2} \mathrm{H}(-/ 0){ }^{4,6,7}$

\section{EXPERIMENTAL DETAILS}

The samples investigated in this study were cut from three different phosphorus $(\mathrm{P})$ doped n-type $\mathrm{Cz}$-Si wafers; their dopant concentrations are presented in Table I. The oxygen and carbon concentrations were about $7 \times 10^{17} \mathrm{~cm}^{-3}$ and below $5 \times 10^{15} \mathrm{~cm}^{-3}$, respectively, as measured by FTIR spectroscopy. The samples were cleaned in a standard RCA solution, and the native oxide layer was dissolved by a dilute HF dip during 10-20 s. Using a shadow mask, circular palladium contacts with a diameter of 1 and $2 \mathrm{~mm}$ and a thickness of $150 \mathrm{~nm}$ were then deposited to form Schottky diodes. The samples were annealed at $300^{\circ} \mathrm{C}$ during $2 \mathrm{~h}$ in nitrogen ambient to diffuse out residual hydrogen that penetrated into the samples during the chemical treatment. Indium-gallium eutectic was used as backside Ohmic contacts. Here, it should be pointed out that the use of palladium Schottky contacts does not appear to influence the defect evolution observed. In a previous study of samples implanted with multiple energy $\mathrm{H}$ ions for analysis by DLTS and MCTS, gold Schottky contacts with a protective aluminum layer were used. ${ }^{9}$ Isochronal annealing of these samples up to $300{ }^{\circ} \mathrm{C}$ revealed identical trends in the defect annealing and formation as those found in the present study.

$\mathrm{H}^{+}$ions were implanted into samples $\mathrm{A}$ and $\mathrm{C} 0-\mathrm{C} 4$ at room temperature with multiple energies to obtain a box-like profile. The projected ion range was selected to cover the depletion region during the subsequent DLTS measurements. A high energy implantation was performed for the B samples such that the $\mathrm{H}^{+}$ions penetrated far beyond the DLTS probing depth, $\sim 48 \mu \mathrm{m}$ versus $4-6 \mu \mathrm{m}$ as estimated by SRIM simulations. ${ }^{12}$ Thus, in the B samples, only hydrogen-free irradiation-induced defects were monitored, and in accordance with previous results, in the literature, ${ }^{13}$ their evolution during annealing can be used as a control to unveil the influence of hydrogen in samples A and C. The implantation

TABLE I. Survey of the samples used.

\begin{tabular}{lcccc}
\hline \hline Sample & $\begin{array}{c}\text { Doping } \\
\left(10^{14} \mathrm{~cm}^{-3}\right)\end{array}$ & $\begin{array}{c}\text { Implantation } \\
\text { energy }(\mathrm{keV})\end{array}$ & $\begin{array}{c}\text { Total dose } \\
\left(10^{11} \mathrm{~cm}^{-2}\right)\end{array}$ & $\begin{array}{c}\text { Heat } \\
\text { treatment }\end{array}$ \\
\hline A & 7.5 & $115-315$ & 1.5 & $30 \mathrm{~min}, 150^{\circ} \mathrm{C}$ \\
B & 7.0 & 2000 & 10 & $30 \mathrm{~min}, 150^{\circ} \mathrm{C}$ \\
C0 & 1.2 & $300-600$ & 0.4 & $30 \mathrm{~min}, 75-125^{\circ} \mathrm{C}$ \\
C1 & & & & $0-860 \mathrm{~min}, 75^{\circ} \mathrm{C}$ \\
C2 & & & & $0-496 \mathrm{~min}, 86^{\circ} \mathrm{C}$ \\
C3 & & & & $0-327 \mathrm{~min}, 90^{\circ} \mathrm{C}$ \\
$\mathrm{C} 4$ & & & $0-165 \mathrm{~min}, 95^{\circ} \mathrm{C}$ \\
\hline \hline
\end{tabular}

parameters for all the studied samples are given in Table I. All the samples were exposed to RT for a few hours (maximum $24 \mathrm{~h}$ ) after the implantation before the first DLTS measurement was undertaken. The samples were then stored in a freezer $\left(-18^{\circ} \mathrm{C}\right)$ between every stage of annealing and analysis. Isochronal and isothermal annealings were carried out at temperatures of $75-150^{\circ} \mathrm{C}$. DLTS and capacitance-voltage (CV) measurements were performed after each annealing stage using a refined version of the setup described in Ref. 14, having a Boonton 7200 capacitance meter, a closed cycle helium cryostat operating in the temperature range from $30 \mathrm{~K}$ to $300 \mathrm{~K}$, and an in-house LabView code for controlling the system. All the DLTS spectra were recorded adopting the GS4 weighting function to obtain high spectral energy resolution. ${ }^{15}$

\section{RESULTS AND DISCUSSION}

The charge carrier profiles of the as-implanted A samples, deduced from the CV measurements, reveal a decrease in the effective doping concentration from $7.5 \times 10^{14}$ to $5.0 \times 10^{14} \mathrm{~cm}^{-3}$ in the $\mathrm{H}^{+}$-implanted region. This is primarily attributed to passivation of the phosphorus donors by hydrogen with formation of electrically inactive phosphorushydrogen (PH) pairs. ${ }^{16} \mathrm{CV}$-profiles of the B sample do not show a significant decrease in the charge carrier concentration after the high-energy implantation. This indicates that the implantation-induced defects alone do not considerably affect the carrier concentration, and the dominant mechanism is the passivation of phosphorus by hydrogen. Based on this observation, we estimate the concentration of $\mathrm{PH}$ pairs in the implanted region of the A samples as $2.5 \times 10^{14} \mathrm{~cm}^{-3}$, while the average hydrogen concentration, estimated from the total implantation dose and the width of the box-like implantation profile, is $3 \times 10^{14} \mathrm{~cm}^{-3}$.

Figure 1 shows DLTS spectra for the samples A and B after implantation and after annealing at $150^{\circ} \mathrm{C}$. The DLTS signal is represented as $2 N_{d} \Delta C / C_{r}$, where $N_{d}$ is the effective doping concentration, $\Delta C$ is the amplitude of the capacitance transient, and $C_{r}$ is the steady-state capacitance at the reverse

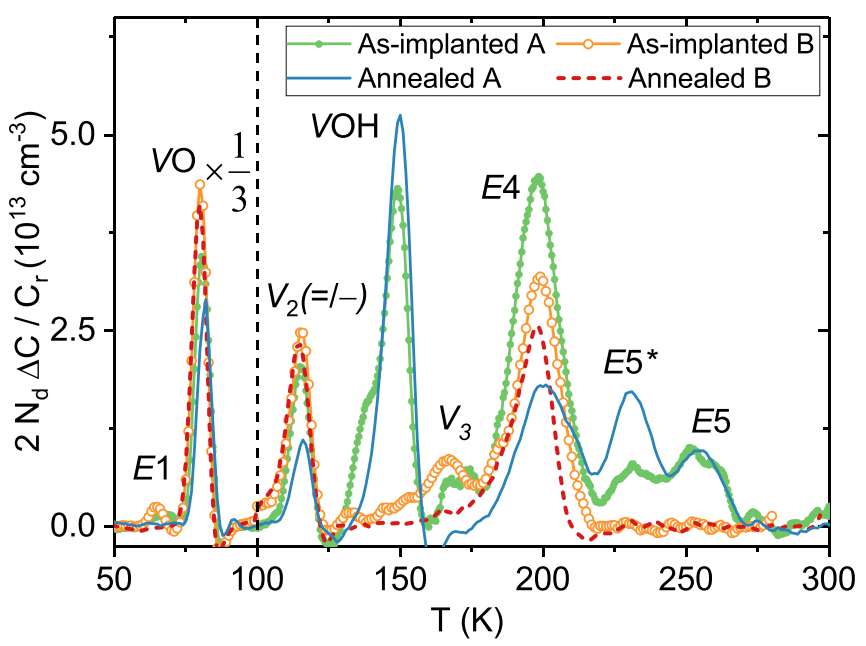

FIG. 1. DLTS spectra for samples A and B before and after annealing at $150{ }^{\circ} \mathrm{C}$ during $30 \mathrm{~min}$. The spectra were acquired with a $(640 \mathrm{~ms})^{-1}$ rate window. 
bias used. In the case of uniform distributions, the concentration of carrier traps, $N_{t}$, equals $2 N_{d} \Delta C / C_{r}$ at the maximum of the corresponding DLTS peak. Before annealing, the DLTS spectrum of sample B contains peaks attributed to vacancy-oxygen pairs $(V \mathrm{O}),{ }^{17} V_{2}(=/-)$, planar-coordinated trivacancies $V_{3}(=/-),{ }^{18}$ and a peak labeled $E 4$ which is an overlap of $V_{2}(-/ 0)$ and $V_{3}(-/ 0) .{ }^{18}$ In addition to these peaks, the corresponding DLTS spectrum for sample A demonstrates several hydrogen-related states. The peak $E 1$ is tentatively attributed to a donor state of complexes involving substitutional carbon, oxygen, and hydrogen. ${ }^{19}$ The spectrum includes also the prominent $V \mathrm{OH}$ peak..$^{6,9,20,21}$ Furthermore, one can notice that the amplitude of $E 4$ relative to that of $V_{2}(=/-)$ and $V_{3}(=/-)$ is considerably higher in sample $\mathrm{A}$ than in sample $\mathrm{B}$, and this is attributed to additional contributions to E4 from hydrogen-related states. That is, in sample A, E4 comprises at least two different contributions, and as mentioned above, $V_{2} \mathrm{H}(-/ 0)$ is a plausible candidate for the H-related one(s). ${ }^{4,6,7}$ In addition, the spectrum of sample A displays two states labeled as $E 5^{*}$ and $E 5$ with approximately equal concentrations. From Arrhenius analysis of DLTS spectra with different rate windows, the activation enthalpies of $E 5^{*}$ and $E 5$ are found to be $0.42 \mathrm{eV}$ and $0.45 \mathrm{eV}$, respectively, with apparent electron capture crosssection (CCS) values of $4 \times 10^{-17}$ and $2 \times 10^{-17} \mathrm{~cm}^{2}$, respectively. The CCS values deduced from direct CCS measurements by the variation of filling pulse duration are equal to $6.0 \times 10^{-18} \mathrm{~cm}^{2}$ and $2.5 \times 10^{-18} \mathrm{~cm}^{2}$ for $E 5^{*}$ and $E 5$, respectively. Thus, the transition entropies $\Delta S / k$ are similar and equal to $\sim 2$ for both $E 5^{*}$ and $E 5$. Furthermore, the electron emission rates of both states do not exhibit any dependence on the electric field. That is, together with their small $\mathrm{CCS}$, these results strongly favor an assignment of $E 5^{*}$ and E5 to acceptor-like transitions.

Upon annealing at $150{ }^{\circ} \mathrm{C}$ for $30 \mathrm{~min}$, the charge carrier profile of sample A recovers, indicating the dissociation of the PH pairs, ${ }^{16}$ while the carrier profile of sample B remains unchanged. $V_{3}$ modifies its configuration from the planarcoordinated to the more stable fourfold one, ${ }^{18}$ and the $V_{3}(-/ 0)$ and $V_{3}(=/-)$ signals disappear in both samples. The amplitudes of the $V \mathrm{O}$ and $V_{2}(=/-)$ signals in sample $\mathrm{B}$ do not change upon the annealing, and it is also important to point out that the amplitudes of the $V_{2}(-/ 0)$ and $V_{2}(=/-)$ peaks become practically equal, as expected for unperturbed/isolated $V_{2}$ centers. In contrast, the spectrum of sample A evolves substantially, indicating the defect interaction with "free" hydrogen, presumably originating from the dissociating PH pairs. ${ }^{16}$ In particular, the presence of hydrogen is manifested by the growth of $\mathrm{VOH}$ and the decrease in $\mathrm{VO}, V_{2}(=/-)$, and E4. E5 does not change significantly, whilst $E 5^{*}$ grows noticeably and is concluded to be a hydrogen-related state. In fact, the amplitude of $E 5^{*}$ exceeds now that of $V_{2}(=/-)$ and $E 5$. It should also be pointed out that simulation of DLTS spectra for the $E 5^{*}$ peak shows an excellent fit, assuming one contributing level only, and no evidence for an overlapping level is found.

The nature of E5 is still not conclusively identified. However, its position in the bandgap is in the same range as the theoretically predicted acceptor level of $V \mathrm{H}^{22}$ and it is reported to anneal out simultaneously with the EPR signal of $V \mathrm{H}^{6,7}$ Furthermore, E5 is only observed in H-implanted samples and not in irradiated ones subjected to subsequent hydrogenation. Hence, the simultaneous presence of hydrogen and mobile primary intrinsic defects, such as $V$, appears to be a necessary condition for $E 5$ formation, corroborating an assignment to $V H$. The $E 5^{*}$ state has recently been reported in Ref. 9 , and it grows after annealing at $75-100{ }^{\circ} \mathrm{C}$ before disappearing at temperatures above $225^{\circ} \mathrm{C}$. A DLTS peak with a similar signature as $E 5^{*}$, labeled $E 10$, was briefly mentioned in Ref. 20 but without any detailed investigation.

In order to gain further understanding of the formation of $E 5^{*}$, the low doped samples $\mathrm{C} 0-\mathrm{C} 4$ (Table I) have been implanted with $\mathrm{H}^{+}$ions. Similar to sample A, the charge carrier profiles of the samples $\mathrm{C} 0-\mathrm{C} 4$ exhibit a decrease after implantation, attributed to the formation of $\mathrm{PH}$ pairs with a concentration of $2 \times 10^{13} \mathrm{~cm}^{-3}$, while the average (uniform) concentration of implanted hydrogen is $5 \times 10^{13} \mathrm{~cm}^{-3}$ in the probed volume.

DLTS spectra of the C0 sample after different stages of $30 \mathrm{~min}$ isochronal annealing are shown in Fig. 2. In comparison to sample $\mathrm{A}$, the $E 1$ amplitude is higher, relative to the other peaks, presumably indicating that residual carbon is more prominent in this material as a trap for hydrogen and implantation-induced defects. The net carrier concentration recovers with annealing, attributed to the dissociation of the PH pairs, and despite the low doping concentration, the evolution of the DLTS spectra is similar to that in sample A. $E 5 *$ grows gradually, and $V \mathrm{O}$ and $V_{2}$ react with "free" hydrogen. There is also a noticeable anticorrelation between the amplitudes of $E 4$ and $E 5^{*}$ upon the annealing.

To analyze the evolution kinetics of $E 5^{*}$ in detail, we have conducted isothermal annealing of the samples $\mathrm{C} 1-\mathrm{C} 4$ in the temperature range of $75-95^{\circ} \mathrm{C}$. The development of the peak amplitudes is presented in Fig. 3. $V O$ and $V_{2}$ react with $\mathrm{H}_{i}$, as inferred from a rapid decrease in $V \mathrm{O}$ (data not shown) and $V_{2}(=/-)$ and an increase in $V \mathrm{OH}$ during the initial stages of annealing. The growth of $E 5^{*}$ is substantially slower, which indicates a different migrating species, for instance $\mathrm{H}_{2}$, or a reaction mechanism involving a barrier.

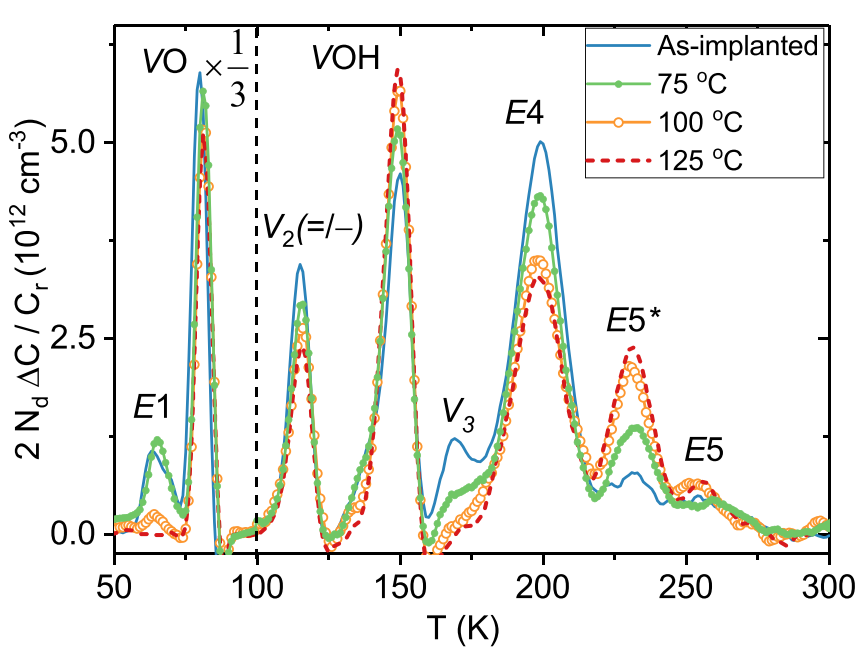

FIG. 2. DLTS spectra of sample C0 after different stages of isochronal annealing, recorded with a $(640 \mathrm{~ms})^{-1}$ rate window. 

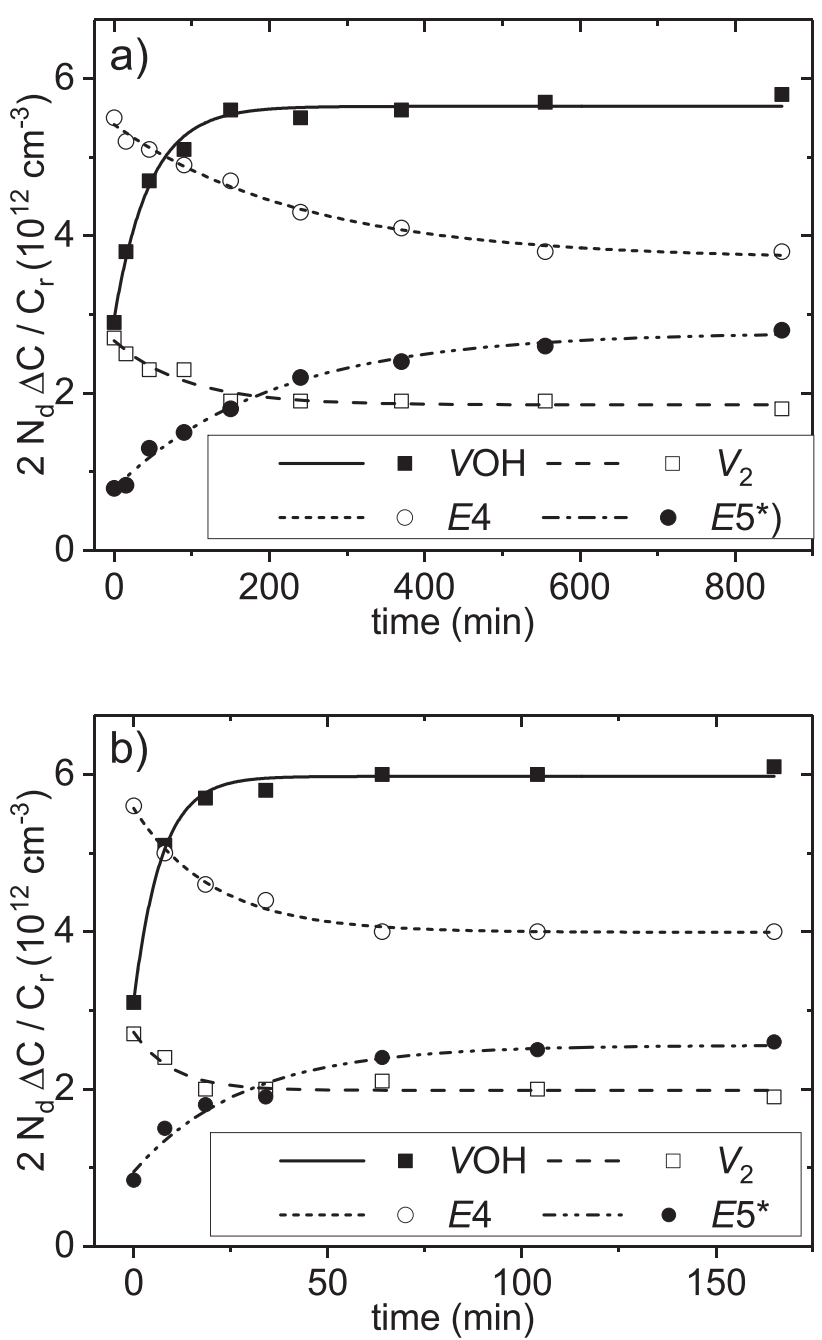

FIG. 3. Amplitudes of different DLTS peaks (symbols) as a function of annealing time at $75^{\circ} \mathrm{C}$ (a) and $95^{\circ} \mathrm{C}$ (b) and the corresponding exponential decay fits (curves).

The formation of $\mathrm{H}_{2}$ in silicon is a somewhat controversial issue, and it is well established that $\mathrm{H}_{2}$ does indeed form, but the exact mechanism is still under debate (see, for instance, Refs. 23 and 24). It is generally accepted that $\mathrm{H}_{i}$ in silicon is an amphoteric, negative- $\mathrm{U}$ defect, and the dominant fractions are either negatively or positively charged depending on the Fermi level position. ${ }^{25}$ Thus, formation of $\mathrm{H}_{2}$ by the direct interaction of two identically charged $\mathrm{H}_{i} \mathrm{~s}$ $\left(\mathrm{H}_{i}+\mathrm{H}_{i} \rightarrow \mathrm{H}_{2}\right)$ has a low probability due to the Coulomb repulsion. It has been suggested, however, that dopants (donors and acceptors) can in fact mediate the formation of $\mathrm{H}_{2}{ }^{26-28}$ In addition, hydrogen is also known to form weakly bound complexes with substitutional carbon $\left(\mathrm{C}_{s}\right)^{29}$ and interstitial oxygen $\left(\mathrm{O}_{i}\right){ }^{30}$ This opens another possible mechanism, where $\mathrm{C}_{s}$ and $\mathrm{O}_{i}$ mediate $\mathrm{H}_{2}$ formation. Besides, the interaction of hydrogen with monovacancies and selfinterstitials, present during the implantation, should not be excluded as a path to form $\mathrm{H}_{2}{ }^{28}$

The aforementioned anti-correlation between $E 5^{*}$ and $E 4$ becomes even more apparent upon the isothermal annealing (Fig. 3), and the increase in the $E 5^{*}$ concentration versus the loss of $E 4$ for the isothermally annealed samples $\mathrm{C} 1-\mathrm{C} 4$ is depicted in Fig. 4. The data points are close to the line $y=x$, evidencing a nearly one-to-one proportionality between the $E 5^{*}$ growth and the $E 4$ loss. As discussed above, $E 4$ contains contributions from at least two levels: $V_{2}(-/ 0)$ and an unknown one, presumably $V_{2} \mathrm{H}(-/ 0){ }^{6,7}$ The contribution from $V_{2}(-/ 0)$ can be estimated from the amplitude of the $V_{2}(=/-)$ peak arising from the same defect center and with no overlapping levels. It should be emphasized that the interaction $V_{2}+\mathrm{H}_{i} \rightarrow V_{2} \mathrm{H}$ does not decrease the $E 4$ peak since $E 4$ is a sum of $V_{2}(-/ 0)$ and $V_{2} \mathrm{H}(-/ 0)$. Thus, the annealing of $E 4$ can be either (i) due to an interaction of $V_{2}$ with another hydrogen species instead $\mathrm{H}_{i}$ or (ii) due to an interaction of $V_{2} \mathrm{H}$ with $\mathrm{H}_{i}$ or other hydrogen species. No correlation is found between the growth of $E 5^{*}$ and the loss of $V_{2}(=/-)$, i.e., $V_{2}$ appears not to be a direct precursor of $E 5^{*}$. Hence, $V_{2} \mathrm{H}(-/ 0)$ seems to be directly associated with the formation of $E 5^{*}$.

The defect evolution upon the isothermal annealing has been fitted by exponential decay to deduce reaction rates (curves in Fig. 3). Figure 5 shows the temperature dependencies of the formation rates for $V \mathrm{OH}$ and $E 5^{*}$. The rates exhibit an Arrhenius behavior, yielding activation energies of $E_{V \mathrm{OH}}=1.05 \mathrm{eV}$ and $E_{E 5^{*}}=1.10 \mathrm{eV}$ and pre-exponential factors $r_{V \mathrm{OH}}$ and $r_{E 5^{*}}$ of around $\sim 10^{11}-10^{12} \mathrm{~s}^{-1}$. The "slow" rate is about a factor of 4 slower within the investigated temperature interval, and the similar activation energies indicate strongly that the diffusing species are the same or very similar in the two cases.

As mentioned above, the origin of the fast kinetics, which governs the annealing of $V \mathrm{O}$ and $V_{2}(=/-)$ and the formation of $V \mathrm{OH}$, is attributed to the $\mathrm{PH}$ dissociation and subsequent diffusion of atomic $\mathrm{H}_{i}$. Hence, based on the correlation plot in Fig. 4 and the Arrhenius analysis of the experimental rates in Fig. 5, it is tempting to ascribe the $E 5^{*}$ formation to the interaction $V_{2} \mathrm{H}+\mathrm{H}_{i} \rightarrow V_{2} \mathrm{H}_{2}$, where the acceptor transition $V_{2} \mathrm{H}_{2}(-/ 0)$ can be associated with $E 5^{*}$. However, one should emphasize that the amplitudes of $\mathrm{VOH}$ and $V_{2}(=/-)$ saturate faster during the isothermal anneals in

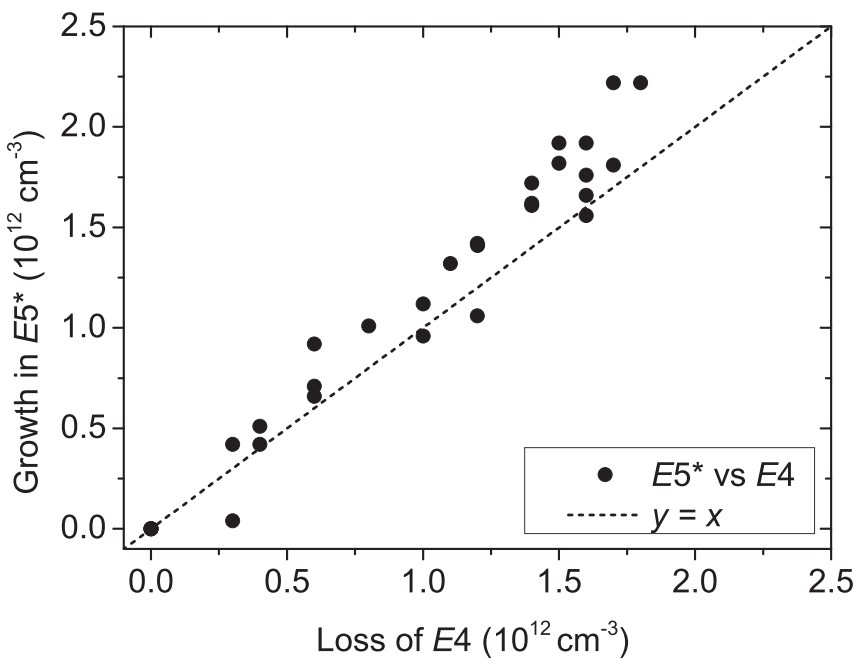

FIG. 4. Correlation between the growth of $E 5^{*}$ and the loss of $E 4$. The values are compiled from the isothermal annealing at $75^{\circ} \mathrm{C}, 86^{\circ} \mathrm{C}, 90^{\circ} \mathrm{C}$, and $95^{\circ} \mathrm{C}$. 


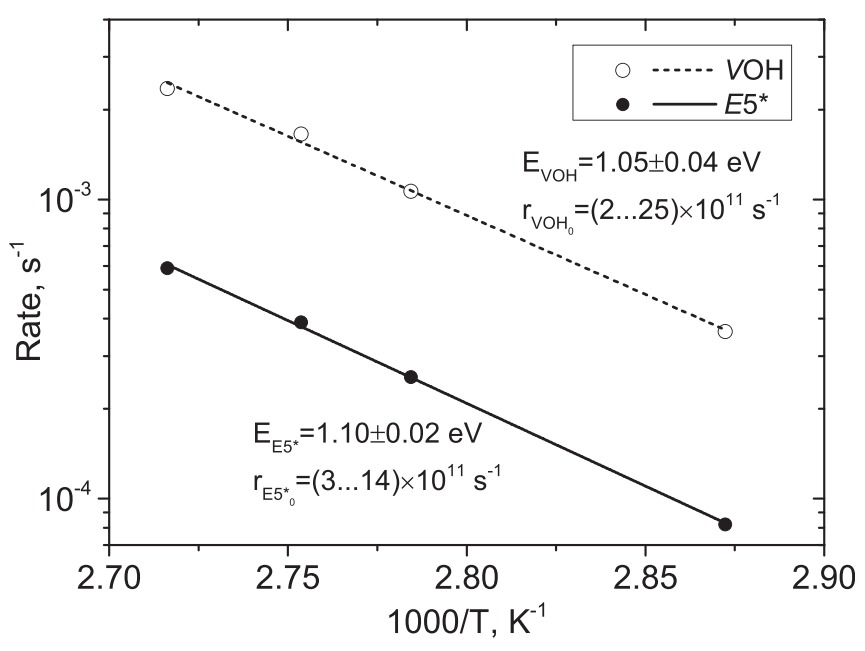

FIG. 5. Arrhenius plot of formation rates for $V \mathrm{OH}$ and $E 5^{*}$ deduced from the isothermal annealing at $75^{\circ} \mathrm{C}, 86^{\circ} \mathrm{C}, 90^{\circ} \mathrm{C}$, and $95^{\circ} \mathrm{C}$.

Fig. 3 than those of $E 4$ and $E 5^{*}$ (the slower kinetics by a factor of 4). A possible explanation of such a delayed kinetics is an intermediate state $\left\{V_{2} \mathrm{H}, \mathrm{H}\right\}$ with a small barrier $(\sim 0.05 \mathrm{eV})$ for the final transition to $V_{2} \mathrm{H}_{2}$.

Based on the kinetics rates, one can rule out the involvement of hydrogen molecules in the $E 5^{*}$ formation since the estimated diffusivity of the hydrogen species involved is several orders higher compared to that obtained by Markevich and Suezawa for the molecules. ${ }^{30}$ Another potential candidate for the slow $E 4$ and $E 5^{*}$ kinetics could be the so-called $\mathrm{H}_{2}{ }^{*}$ dimer, ${ }^{31}$ which can result in the interaction $V_{2} \mathrm{H}+\mathrm{H}_{2}^{*} \rightarrow V_{2} \mathrm{H}_{3}$. The diffusivity of $\mathrm{H}_{2} *$ is poorly investigated but can be anticipated to be slower than that of $\mathrm{H}_{i}$, although it may not seem probable that the difference is only a factor of 4 .

\section{CONCLUSIONS}

The $V_{2}$ evolution in hydrogen-implanted Czochralskigrown silicon samples upon isochronal and isothermal annealing has been studied by DLTS. An acceptor-like electronic state, labeled $E 5^{*}$, with the energy position $E_{c}-0.42 \mathrm{eV}$ has been observed. The $E 5^{*}$ center is present only in the hydrogen-enriched material, evidencing its hydrogen-related nature. $E 5^{*}$ anticorrelates, in a close one-to-one ratio, with a hydrogen-related complex, presumably $V_{2} \mathrm{H}$, which contributes to the $V_{2}$-containing $E 4$ peak. Isothermal heat treatment reveals slower formation kinetics for $E 5^{*}$, by a factor of 4 , compared to the interactions of $V \mathrm{O}$ and $V_{2}$ with $\mathrm{H}_{i}$. It implies the presence of a small barrier $(\sim 0.05 \mathrm{eV})$ for the interaction of $\mathrm{H}_{i}$ with $V_{2} \mathrm{H}$ or another slower diffusing hydrogen species being involved in the formation of $E 5^{*}$.

\section{ACKNOWLEDGMENTS}

This work was performed within "The Norwegian Research Centre for Solar Cell Technology" (Project No.
460976), a Centre for Environment-friendly Energy Research cosponsored by the Norwegian Research Council and research and industry partners in Norway. The Research Council of Norway is thanked for the support to the Norwegian Microand Nano-Fabrication Facility, NorFab (Project No. 245963).

${ }^{1}$ G. D. Watkins and J. W. Corbett, Phys. Rev. 138, A543 (1965).

${ }^{2}$ E. V. Monakhov, A. Ulyashin, G. Alfieri, A. Y. Kuznetsov, B. S. Avset, and B. G. Svensson, Phys. Rev. B -Condens. Matter Mater. Phys. 69, 153202 (2004).

${ }^{3}$ P. Stallinga, P. Johannesen, S. Herstro, K. B. Nielsen, and B. B. Nielsen, Phys. Rev. B 58, 3842 (1998).

${ }^{4}$ J. Coutinho, V. J. B. Torres, R. Jones, S. Oberg, and P. R. Briddon, J. Phys. Condens. Matter 15, S2809 (2003).

${ }^{5}$ B. G. Svensson, B. Mohadjeri, A. Hallen, J. H. Svensson, and J. W. Corbett, Phys. Rev. B 43, 2292 (1991).

${ }^{6}$ K. B. Nielsen, L. Dobaczewski, K. Goscinski, R. Bendesen, O. Andersen, and B. B. Nielsen, Phys. B Condens. Matter 273-274, 167 (1999).

${ }^{7}$ P. Lévêque, A. Hallén, B. G. Svensson, J. Wong-Leung, C. Jagadish, and V. Privitera, Eur. Phys. J. Appl. Phys. 23, 5 (2003).

${ }^{8}$ J. H. Bleka, H. Malmbekk, E. V. Monakhov, B. G. Svensson, and B. S. Avset, Phys. Rev. B -Condens. Matter Mater. Phys. 85, 85210 (2012).

${ }^{9}$ I. L. Kolevatov, F. Herklotz, V. Bobal, B. G. Svensson, and E. V. Monakhov, Solid State Phenom. 242, 163 (2015).

${ }^{10}$ G. Alfieri, E. V. Monakhov, B. S. Avset, and B. G. Svensson, Phys. Rev. B 68, 233202 (2003).

${ }^{11}$ E. V. Monakhov, J. Wong-Leung, A. Y. Kuznetsov, C. Jagadish, and B. G. Svensson, Phys. Rev. B 65, 245201 (2002).

${ }^{12}$ J. F. Ziegler, M. D. Ziegler, and J. P. Biersack, Nucl. Instrum. Methods Phys. Res., Sect. B 268, 1818 (2010).

${ }^{13}$ E. V. Monakhov and B. G. Svensson, in Silicon, Germanium and their Alloys: Growth, Defects, Impurities, Nanocrystals, edited by G. Kissinger and S. Pizzini (CRC Press, 2014), pp. 255-288.

${ }^{14}$ B. G. Svensson, K. H. Rydén, and B. M. S. Lewerentz, J. Appl. Phys. 66, 1699 (1989).

${ }^{15}$ A. A. Istratov, J. Appl. Phys. 82, 2965 (1997).

${ }^{16}$ J. Zhu and N. M. Johnson, Phys. Rev. B 41, 12354 (1990).

${ }^{17}$ S. D. Brotherton and P. Bradley, J. Appl. Phys. 53, 5720 (1982).

${ }^{18}$ V. P. Markevich, A. R. Peaker, S. B. Lastovskii, L. I. Murin, J. Coutinho, A. V. Markevich, V. J. B. Torres, P. R. Briddon, L. Dobaczewski, E. V. Monakhov, and B. G. Svensson, Phys. B Condens. Matter 404, 4565 (2009).

${ }^{19}$ M. Yoneta, Y. Kamiura, and F. Hashimoto, J. Appl. Phys. 70, 1295 (1991).

${ }^{20}$ K. Irmscher, H. Klose, and K. Maass, J. Phys. C Solid State Phys. 17, 6317 (1984).

${ }^{21}$ H. Malmbekk, L. Vines, E. V. Monakhov, and B. G. Svensson, Solid State Phenom. 178-179, 192 (2011).

${ }^{22}$ H. Xu, Phys. Rev. B 46, 1403 (1992).

${ }^{23}$ A. W. R. Leitch, V. Alex, and J. Weber, Phys. Rev. Lett. 81, 421 (1998).

${ }^{24}$ V. V. Voronkov and R. Falster, Phys. Status Solidi Basic Res. 254, 1600779 (2017)

${ }^{25}$ K. J. Chang and D. J. Chadi, Phys. Rev. B 40, 11644 (1989).

${ }^{26}$ L. Korpas, J. W. Corbett, and S. K. Estreicher, Phys. Rev. B 46, 12365 (1992).

${ }^{27}$ Z. N. Liang, P. J. H. Denteneer, and L. Niesen, Phys. Rev. B 52, 8864 (1995).

${ }^{28}$ R. Jones, B. J. Coomer, J. P. Goss, B. Hourahine, and A. Resende, Solid State Phenom. 71, 173 (2000).

${ }^{29}$ R. Stübner, V. Kolkovsky, and J. Weber, J. Appl. Phys. 118, 55704 (2015).

${ }^{30}$ V. P. Markevich and M. Suezawa, J. Appl. Phys. 83, 2988 (1998).

${ }^{31}$ J. D. Holbech, B. Bech Nielsen, R. Jones, P. Sitch, and S. Berg, Phys. Rev. Lett. 71, 875 (1993). 